\title{
Transverse Bragg-reflector injection lasers
}

\author{
J. B. Shellan, W. Ng, P. Yeh, and A. Yariv \\ California Institute of Technology, Pasadena, California 91125
}

\section{A. Cho}

Bell Laboratories, Murray Hill, New Jersey 07974

Received February 14, 1978

\begin{abstract}
A GaAs-GaAlAs injection laser has been tested that confines light in the lateral dimension (normal to junction plane) by a multilayer Bragg reflector. In the past, light has been confined as a result of the higher-index guiding region and resulting evanescent fields.
\end{abstract}

Recently passive waveguide structures have been grown by molecular beam epitaxy, which confines light in the transverse direction as a result of a multilayer Bragg reflector. ${ }^{1}$ Because of the Bragg reflector, light can actually be confined in a guiding region that in a conventional structure would not allow confinement, either because the index of the guiding layer is lower than the surrounding material or because the "guiding" layer is too thin.2 A symmetric guide structure has no cutoff thickness, but a conventional asymmetric guide will not support a mode if the guiding layer is less than a cutoff thickness $t_{c}$. The transverse Bragg reflector laser will support a mode for extremely thin active layers.

\section{Theory}

Figure 1 shows the geometry of a typical structure as well as the intensity profiles of confined radiation. The amplitude of the light in the $l$ th layer can be described by $A_{l} \exp \left(i \mathbf{k}_{l} \cdot \mathbf{r}\right)$, where $A_{l}$ is an amplitude factor and $k_{l}$ is the wavevector in that layer, $\left|k_{l}\right|=\left(2 \pi / \lambda_{0}\right) n_{l}, n_{l}$ being the index of refraction, $\lambda_{0}=$ wavelength of light in vacuum.

Clearly for coherent interaction with the reflector layers and maximum confinement, we require

$$
k_{l x}=\frac{\pi}{2 t_{l}} p,
$$

where $t_{l}$ is the thickness of the $l$ th layer and $p$ is an integer. Thus we see that a mode that propagates with a particular frequency $\omega$ and an appropriate $k_{z} \equiv \beta$ will satisfy Eq. (1). Other $\omega-k_{z}$ combinations will not and thus will have higher losses. In fact it can be shown from the coupled-mode approximation that near the conditions satisfied by Eq. (1) the attenuation or loss constant $\alpha$ due to the finite number of reflector layers is given by

$$
\begin{gathered}
\alpha=\left[\left(1-\frac{\delta^{2}}{\eta^{2}}\right) \frac{\kappa}{\beta} e^{-\left(\eta^{2}-\delta^{2}\right)^{1 / 2} L}\right] \\
\div\left[t+\frac{2 \eta-\frac{2 \delta^{2}}{n}+\frac{\delta \kappa}{n}}{\kappa^{2}+4\left(\eta^{2}-\delta^{2}\right)}\right. \\
\left.+\frac{1}{2}\left(\frac{1}{\sqrt{\eta^{2}-\delta^{2}}}+\frac{\sqrt{\eta^{2}-\delta^{2}}}{\eta k_{a x}}-\frac{2 \delta}{\eta k_{g x}}+\frac{1}{k_{a x}}\right)\right],
\end{gathered}
$$

where

$$
\begin{aligned}
& \kappa=2 \pi / \Lambda ; \\
& \Lambda=\text { period of Bragg reflector; } \\
& \beta \equiv k_{z}, k_{i}=\left(2 \pi / \lambda_{0}\right) n_{i}, \quad i=1,2, g, a ; \\
& k_{i x}=\sqrt{{k_{i}}^{2}-\beta^{2}}, \quad i=1,2, g, \quad k_{a x}=\sqrt{\beta^{2}-k_{a}^{2}} ; \\
& \eta=\left|\frac{k_{1 x}-k_{2 x}}{k_{1 x}+k_{2 x}}\right| \frac{2}{\Lambda} ; \\
& \delta=\text { phase mismatch }=\frac{1}{\kappa}\left[\frac{k_{1}^{2}+k_{2}^{2}}{2}-\beta^{2}-\left(\frac{\kappa}{2}\right)^{2}\right] ;
\end{aligned}
$$

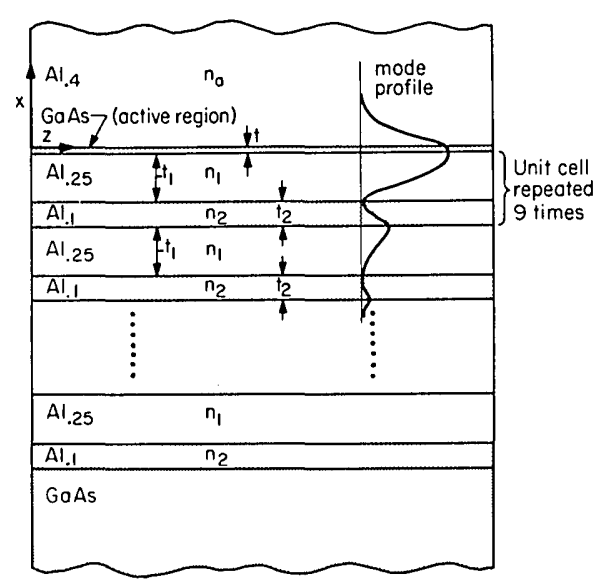

Fig. 1. The geometry of a transverse Bragg reflector laser with intensity profile of confined radiation. 


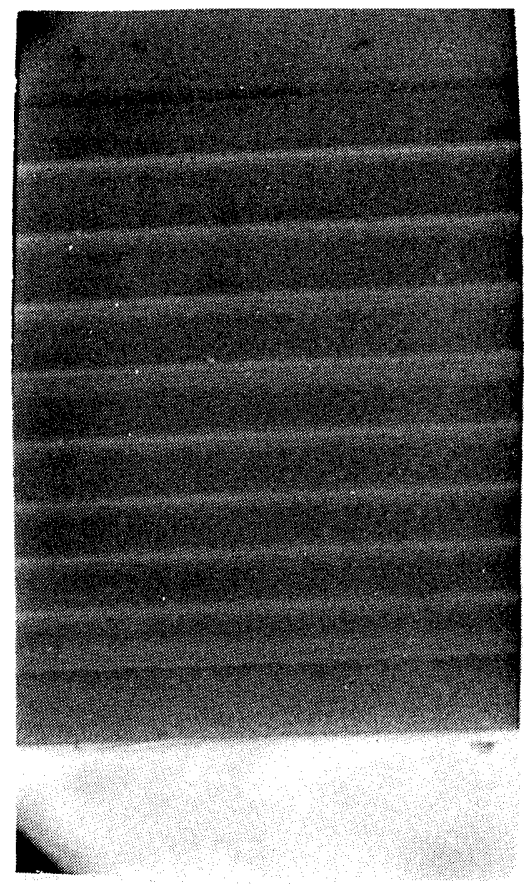

Fig. 2. SEM picture of the injection laser grown by LPE.

$L=$ length of Bragg reflector region $=N \Lambda$;

$N=$ number of periods;

$t=$ thickness of active region.

The loss constant $\alpha$ accounts for losses into the substrate $(\mathrm{GaAs})$. The eigenvalue $\beta$ is determined for the low-loss structures by

$$
\tan k_{g x} t=\frac{k_{a x}}{k_{g x}}
$$

\section{Fabrication}

An SEM picture of the injection laser is shown in Fig. 2. It was grown by liquid-phase epitaxy and consists of a GaAs substrate followed by nine layers of $\mathrm{Al}_{0.1} \mathrm{Ga}_{0.9} \mathrm{As}-\mathrm{Al}_{0.25} \mathrm{Ga}_{0.75} \mathrm{As}$ reflector material, a pure $\mathrm{GaAs}$ active region, and a low-index $\mathrm{Al}_{0.4} \mathrm{Ga}_{0.6}$ As cap. The GaAs active layer is $500 \AA$ thick, and it should be noted that the $\mathrm{Al}$ in all the reflector layers is necessary to avoid excessive absorption in these passive layers. The thicknesses of the $t_{1}$ and $t_{2}$ layers are 3900 and 2200 $\AA$, to provide maximum reflectivity near $\lambda_{0}=8900 \AA$. From Eq. (2), the loss constant for this structure (at $\delta$ $=0$ ) should be $\alpha \simeq 0.25 / \mathrm{cm}$. Thus the gain required for oscillation is $\gamma \simeq \alpha+\alpha^{\prime}+(1 / l) \ln \left(1 / r^{2}\right)^{3}$, where $l=$ length of laser $\simeq 350 \mu \mathrm{m}, r=$ reflectivity $\simeq 0.56$. $\alpha^{\prime}$ is the loss constant due to free-carrier absorption within the recombination region and absorption outside of the recombination region. $\alpha^{\prime} \simeq 20 / \mathrm{cm}$. ${ }^{4}$ Thus $\gamma \simeq 50 / \mathrm{cm}$, and the losses into the substrate due to nine periods are insignificant compared with the Fabry-Perot output losses and absorption. Threshold for lasing occurred at about $6 \mathrm{kA} / \mathrm{cm}^{2}$. The thickness of the active region was chosen as $500 \AA$ to be below cutoff for conventional guiding. Thus, any lasing must be a result of Bragg confinement and Bragg guiding.

\section{Experiment}

Figure 3 shows the longitudinal mode structure of the laser, while Fig. 4 shows the transverse mode intensity. The mode spacing in Fig. 3 corresponds to the FabryPerot spacing of longitudinal modes in a cavity of the length of the laser. Lasing did not occur at $8900 \AA$, most likely because of small amounts of $\mathrm{Al}$ in the atmosphere. The transverse-mode intensity was measured by placing a 43X microscope objective next to the laser, imaging the near field onto a rotating mirror, which in turn reflected the light through a $30-\mu \mathrm{m}$ slit and onto a photomultiplier. The voltage controlling the mirror rotation was used as the $x$ input to an $x-y$ recorder, while the photomultiplier output was fed into the $y$ input. The distance between peaks is about $0.6 \mu \mathrm{m}$.

\section{Conclusion}

We have demonstrated in an injection laser the confinement of light in the lateral direction as a result of coherent reflection from a layered medium. The losses for this guiding differ for various transverse modes, and thus mode discrimination in the transverse direction may be possible based on the confinement factor for the mode in question. It is expected that this selectivity

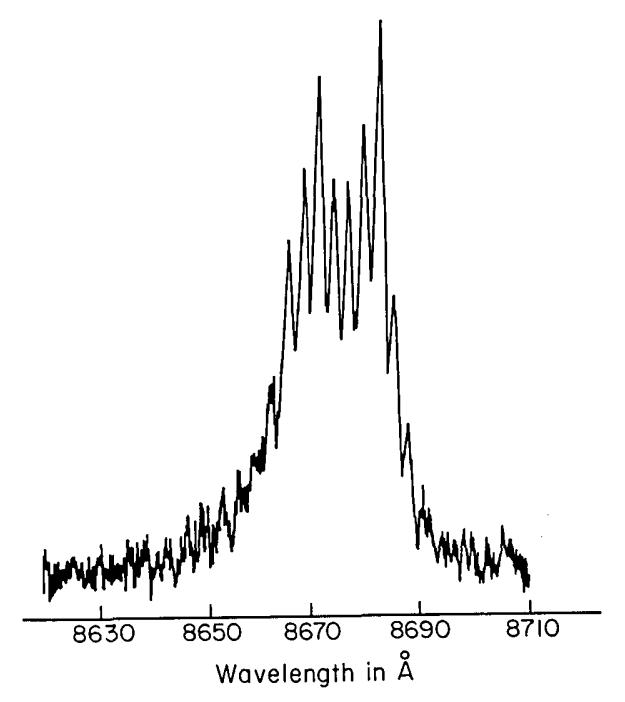

Fig. 3. Longitudinal-mode structure indicating Fabry-Perot spacing of modes.

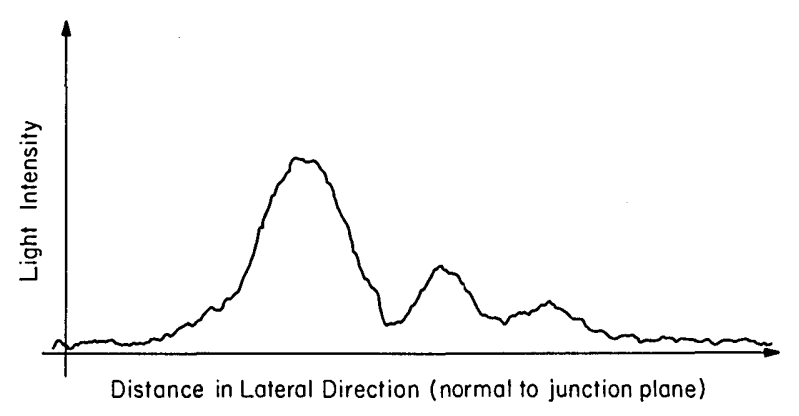

Fig. 4. Transverse-mode intensity normal to the junction plane as measured by experiment. 
would be greater for a structure with fewer reflector layers than the device tested, so that other loss mechanisms are comparable with the substrate radiation losses given by Eq. (2).

The research reported in this Letter was supported by the National Science Foundation (Optical Communication Program) and the Air Force Office of Scientific Research.

J. B. Shellan is a Fannie and John Hertz Foundation Doctoral Fellow.
P. Yeh is currently at Rockwell International Science Center, Thousand Oaks, California 91360.

\section{References}

1. A. Y. Cho, A. Yariv, and P. Yeh, Appl. Phys. Lett. 30, 471 (1977).

2. P. Yeh, A. Yariv, and C. S. Hong, J. Opt. Soc. Am. 67, 423 (1977).

3. M. B. Panish, Proc. IEEE 64, 1512 (1976).

4. H. Kressel and M. Ettenberg, J. Appl. Phys. 47, 3533 (1976). 